\title{
Cannulation of Occluded Inferior Petrosal Sinuses for the Transvenous Embolization of Cavernous Sinus Dural Arteriovenous Fistulas: Usefulness of a Frontier-Wire Probing Technique
}

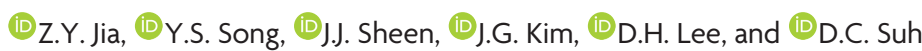

\begin{abstract}
BACKGROUND AND PURPOSE: Pursuing an alternative access route for transvenous embolization of cavernous sinus dural arteriovenous fistulas can be challenging in patients with an occluded inferior petrosal sinus. We found that cannulation of even a completely occluded inferior petrosal sinus is feasible, especially when using a standard hydrophilic-polymer-jacketed 0.035 -inch guidewire as a frontier-wire for probing.
\end{abstract}

MATERIALS AND METHODS: From 2002 to 2017, the frontier-wire technique was tried in 52 patients with occluded inferior petrosal sinuses for transvenous embolization of cavernous sinus dural arteriovenous fistulas at our center. Technical success was defined as access into the affected cavernous sinus compartment with a microcatheter through the occluded inferior petrosal sinus and deployment of at least 1 coil. The complications and treatment outcomes were analyzed.

RESULTS: The frontier-wire technique was applied in 52 patients with 57 occluded inferior petrosal sinuses ( 52 ipsilateral and 5 contralateral inferior petrosal sinuses). Technical success rates were $80.8 \%$ (42/52) of patients and $73.7 \%$ (42/57) of inferior petrosal sinuses. Alternative transvenous routes were used in 3 patients, and transarterial access was used in 7 patients. Complete embolization of fistulas was achieved in $82.2 \%$ (37/45) of patients in the transvenous embolization group and in $14.3 \%(1 / 7)$ of patients in the transarterial group. No procedure-related morbidity or mortality was observed.

CONCLUSIONS: Transvenous embolization of cavernous sinus dural arteriovenous fistulas, even through a completely occluded inferior petrosal sinus, is feasible. The difficulty of passing the microcatheter can be minimized by prior probing of the occluded inferior petrosal sinus using a standard 0.035-inch guidewire; the trace of the guidewire on the roadmap image serves as a guide for microcatheter navigation through the inferior petrosal sinus on fluoroscopy.

ABBREVIATIONS: $C S=$ cavernous sinus; CSDAVF = cavernous sinus dural arteriovenous fistula; IJV = internal jugular vein; IPS = inferior petrosal sinus; $T$ VVE = transvenous embolization

C avernous sinus dural arteriovenous fistula (CSDAVF) is defined as an abnormal arteriovenous connection involving the dura mater within or near the walls of the cavernous sinus (CS). ${ }^{1}$ Due to multiple small feeding arteries to CS fistulas and some of the feeders supplying the vasa nervorum of cranial nerves, transarterial embolization of CSDAVFs is viewed as a low-cure and

Received August 13, 2018; accepted after revision September 18.

From the Department of Radiology and Research Institute of Radiology (Z.Y.J., Y.S.S., J.J.S., J.G.K., D.H.L., D.C.S.), Asan Medical Center, University of Ulsan College of Medicine, Seoul, Korea; and Department of Radiology (Z.Y.J., Y.S.S.), The First Affiliated Hospital with Nanjing Medical University, Nanjing, Jiangsu Province, China.

Z.Y. Jia and Y.S. Song contributed equally to this work.

Please address correspondence to Deok Hee Lee, MD, PhD, Department of Radiology and Research Institute of Radiology, Asan Medical Center, University of Ulsan College of Medicine, 88, Olympic-ro 43-gil, Songpa-gu, Seoul 138-736, Korea; e-mail:dhlee@amc.seoul.kr

http://dx.doi.org/10.3174/ajnr.A5868 high-risk treatment approach. ${ }^{2,3}$ Typically, transvenous embolization (TVE) confers better outcomes and is still the standard treatment for CSDAVFs. ${ }^{3,4}$

The inferior petrosal sinus (IPS) provides a relatively direct and shortest route from the internal jugular vein (IJV) to the CS and is the most commonly used transvenous approach to obliterate the compartments of the affected sinus or, optimally, the shunt hole itself. ${ }^{5}$ However, this route is occasionally thrombosed or collapsed and angiographically invisible at the time of treatment. ${ }^{2}$ Although alternative venous approaches have been reported, including the superior petrosal sinus, ${ }^{6}$ the facial vein, ${ }^{1,2}$ and direct exposure of the superior ophthalmic vein, ${ }^{7}$ the ipsi- or contralateral IPS is still considered the first-line access route for approaching the CS, even in the case of occlusion. ${ }^{2,4}$

Due to the large difference in the diameters of the IJV and the IPS and the angulated course of the IPS itself, it is not easy to find the ostium of the occluded IPS directly with a microguidewire and 
to advance the microguidewire to probe the occluded IPS into the CS. On the basis of our previous experience with cannulating the IPS for petrosal sinus blood sampling with regular diagnostic catheters (4F, Glidecath; Terumo, Tokyo, Japan) and 0.035-inch, polymer-jacketed guidewires (Radifocus; Terumo; or Crescendo; Sungwon Medical, Chungbuk, Korea), we thought that the regular 0.035-inch guidewire would show better performance because it had better controllability and could provide more support than a microguidewire. Once the tip of the guidewire is engaged in the orifice of the IPS, the guidewire can be advanced against the resistance from the collapsed IPS. However, the potential complications such as a venous injury in the posterior fossa may worry some operators in clinical practice.

The purpose of this study was to report our experience with the frontier-wire technique using a 0.035 -inch hydrophilic guidewire for cannulating occluded IPSs in patients undergoing TVE of CSDAVFs. The efficacy and safety of the technique are described.

\section{MATERIALS AND METHODS Study Population}

We retrospectively reviewed our institutional data base (Asan Medical Center) of patients who underwent endovascular treatment for CSDAVFs from January 2002 to September 2017 ( $n=$ 126). Feeding arteries, fistula locations, and their venous drainage patterns were evaluated by reviewing diagnostic cerebral angiography before endovascular treatment. Two biplane angiography systems (Artis zee; Siemens, Erlangen, Germany) were used for image acquisition and endovascular treatment.

Among those patients with CSDAVFs, the ones included in this study met the following criteria: 1) angiographic complete occlusion of the IPS either ipsilateral to the fistula side or bilateral IPS occlusions, and 2) primary treatment attempted with TVE via the occluded IPS, which had been probed with a 0.035 -inch guidewire. Exclusion criteria for this study were the following: 1) angiographic patency of the ipsilateral IPS, or 2) catheterization of the occluded IPS with a microguidewire and microcatheter, without prior probing with a 0.035 -inch guidewire.

A total of 52 patients met the inclusion criteria. General characteristics of the cohort are summarized in the Table. Therapeutic alternatives were discussed as a multidisciplinary decision-making process. Informed procedural consent had been obtained from patients before they underwent endovascular treatment. Institutional review board approval was obtained for this retrospective study.

\section{Endovascular Treatment}

The procedures were performed with the patient under general anesthesia. During the procedure, each patient received 50-80 IU $/ \mathrm{kg}$ of intravenous heparin to attain an activated clotting time of approximately 250-300 seconds. An additional 1000 IU of heparin per hour was given to maintain the activated clotting time. Usually, a 4F angiography catheter was introduced into the external or internal carotid artery by way of main feeding arteries for the control angiographies during the TVE approach, and a $5 \mathrm{~F}$ $(n=8)$ or $6 \mathrm{~F}(n=44)$ guiding catheter (Envoy, Codman Neurovascular, Raynham, Massachusetts) was navigated into the IJV for TVE. If cannulation of the occluded IPS failed, alternative trans-
Characteristics of the 52 patients in the study population

\begin{tabular}{lc}
\hline \multicolumn{1}{c}{ Characteristics } & (No.) (\%) \\
\hline Age (mean) (yr) & $59.1 \pm 10.7$ \\
Sex & \\
$\quad$ Male & $12(23.1)$ \\
Female & $40(76.9)$ \\
Type & \\
Idiopathic & $50(96.2)$ \\
Traumatic & $2(3.8)$ \\
Surgical & $0(0.0)$ \\
Symptoms & \\
Ocular & $47(90.4)$ \\
Cranial nerve palsy & $23(44.2)$ \\
Headache & $15(28.8)$ \\
Tinnitus & $11(21.2)$ \\
Cognard classification & \\
Ila & $25(48.1)$ \\
Ila +llb & $13(25.0)$ \\
III & $2(3.8)$ \\
IV & $12(23.1)$ \\
Side of shunts & \\
Right & $28(53.8)$ \\
Left & $24(46.2)$ \\
Contralateral-IPS occlusion & $42(80.8)$ \\
\hline
\end{tabular}

venous routes such as the contralateral IPS, ipsilateral superior petrosal sinus, or facial vein were considered. If transarterial embolization was needed, the $4 \mathrm{~F}$ catheter was changed into a $6 \mathrm{~F}$ guiding

catheter for the arterial feeder approach. The side arms of the angiographic catheter and the guiding catheter were flushed continuously with pressurized and heparinized normal saline. The percutaneous ophthalmic vein approach was only used if all other approaches were infeasible.

\section{Probing Technique with a 0.035-Inch Hydrophilic Guidewire (Frontier-Wire Technique)}

The technical steps of the procedure are as follows (Fig 1): 1) Advance the guiding catheter inferior to the jugular bulb under the guidance of the jugular venographic roadmap and turn the tip of the curved guiding catheter anteromedially to face the orifice of the occluded IPS. Sometimes a thronelike structure can be seen. 2) By means of the 0.035-inch guidewire, probe the orifice of the IPS on the anteromedial wall of the IJV, even without any visible structure at the orifice. 3) Once the orifice is selected, gently rotate and advance the guidewire through the occluded IPS along the imaginary anatomic course until there is any resistance or blocking. 4) Obtain a new jugular venographic roadmap through the guiding catheter while the guidewire is in situ. 5) Pull back and remove the guidewire to leave a bright guidewire track as part of the roadmap image. 6) Referring to that roadmap information, insert a microcatheter (Excelsior 1018, Stryker, Kalamazoo, Michigan; Headway 17, MicroVention, Tustin, California; Echelon 10, Covidien, Irvine, California; or Prowler Plus, Codman \& Shurtleff, Raynham, Massachusetts) over a microguidewire (Traxcess 14, MicroVention; Transend 14, Stryker; or Synchro 14, Stryker) along the bright track and advance it further into the CS sac. 7) Inject a small amount of contrast media through the microcatheter to confirm that the microcatheter tip is in the target compartment right before the coil packing.

The following anatomic and technical points are helpful (Fig 


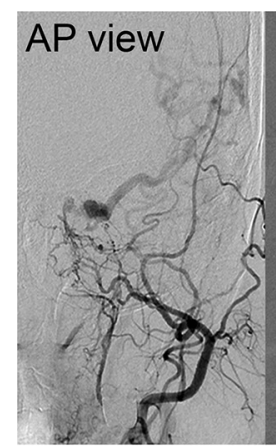

A
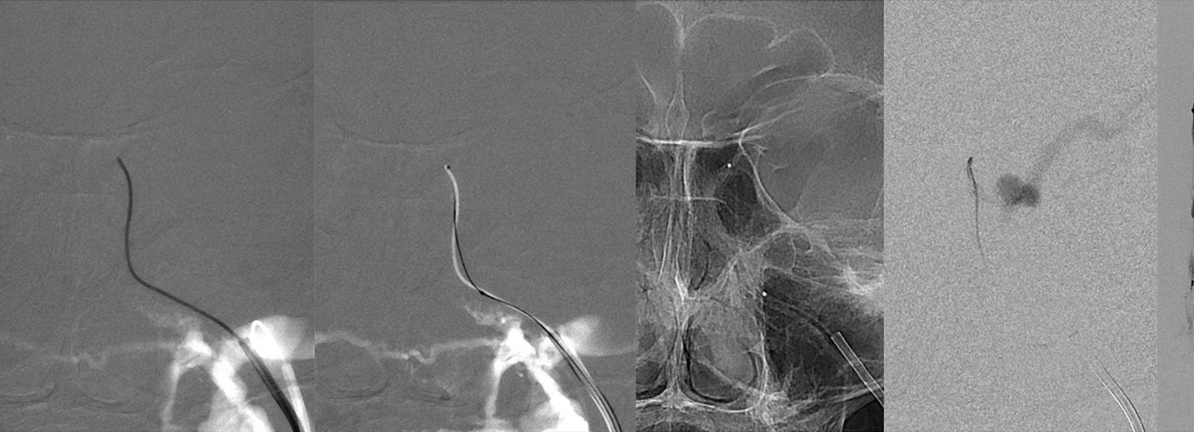

C E

G

I

K

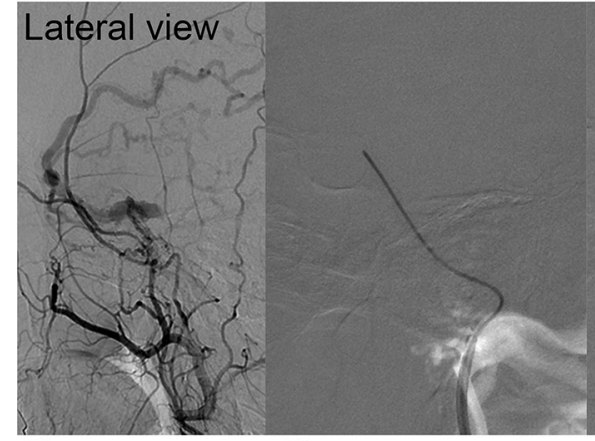

B
D
F

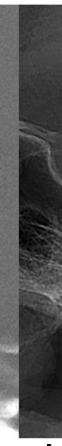

$\mathrm{H}$

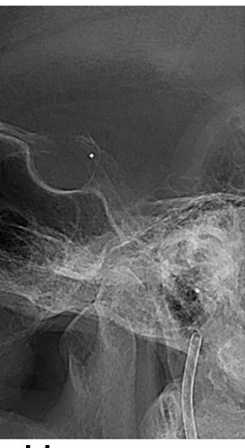

$\mathrm{H}$

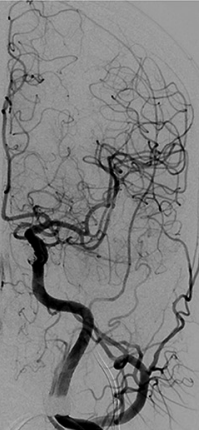

FIG 1. A representative case showing the probing technique (the patient presented with left proptosis, chemosis for 10 months, and development of headache and nausea in the preceding month). $A$ and $B$, Left CSDAVFs shown by left external carotid artery angiography. Note ipsilateral-IPS occlusion and main venous drainage of the superficial middle cerebral vein. The fistula was supplied by multiple dural branches of both the ICA and external carotid artery. $C$ and D, With a $5 \mathrm{~F}$ guiding catheter positioned in the left IJV, the IPS was selected and probed with a 0.035 -inch guidewire to the area of the CS. $E$ and $F, A$ new roadmap was obtained with retrograde injection of contrast through the guiding catheter. A white mark was left on the roadmap by pulling back the guidewire. A microguidewire and microcatheter were introduced into the IPS and advanced into the CS under the guidance of the roadmap. $G$ and $H$, Note the microcatheter tip relative to bony structures. I and J, DSA images demonstrate gentle hand injection of the left CS, with subsequent filling of left middle cerebral vein. $K$ and $L$, Transvenous coil embolization was performed, and no residual fistula was observed on completion angiography. AP indicates anteroposterior.
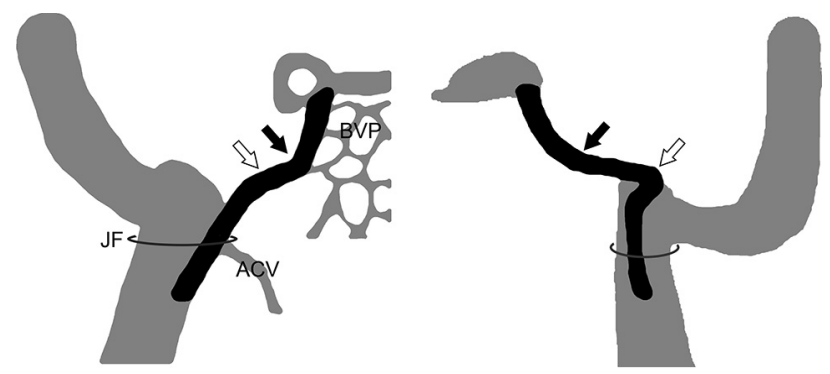

FIG 2. Illustration of the IPS in an anteroposterior (left) and lateral (right) view. The IPS mostly arises from the anteromedial wall of the IJV, lower than the level of the JF. In general, the IPS runs anterior, medial, and cephalad; however, the course is not simply straight, and usually, 2 curves can be identified. There is a lower curve where the IPS leaves the jugular foramen, appearing as a smooth course on the anteroposterior view, but as a sharp angle on the lateral view (open arrows). There is an upper curve where the IPS runs in the inferior petrosal sulcus (closed arrows). Around this curve, there are many anastomotic channels between the IPS and the basilar venous plexus (anteroposterior view, closed arrow). JF indicates jugular foramen; $\mathrm{ACV}$, anterior condylar vein.

2) to facilitate safe and effective probing: 1) The IPS arises from the anteromedial wall of the IJV, usually lower than the level of the jugular foramen. 2) There are 2 obvious curves in the course of the IPS (Fig 2), and the guidewire tip should be turned to adapt to the course at these 2 curves. 3 ) As described previously, ${ }^{4} 2$ methods can be used to reinforce the guidewire: Either insert the guid- ing catheter as close as possible to the IPS orifice, or by means of the coaxial technique, introduce an intermediate $4 \mathrm{~F}$ catheter into the orifice of the IPS following the guidewire. In contrast to the patent sinus situation, the diagnostic catheter most often cannot be advanced deep into the occluded IPS. 4) A 6F guiding catheter can accommodate a 0.035 -inch guidewire and a microcatheter (Headway 17, Excelsior 1018, or Echelon 10) in parallel. Therefore, the 0.035 -inch guidewire may be left in the IPS partially to direct the guiding catheter toward the orifice of the IPS, which can facilitate the introduction of the microcatheter into the IPS. 5) After successful passage of the 0.035 -inch guidewire through the collapsed IPS, successive microguidewire navigation is usually accomplished without difficulty.

\section{Technical, Imaging, and Clinical Outcomes}

The anatomic level of advancement of the 0.035 -inch guidewire along the imaginary course of the occluded IPS was classified as proximal or distal using the upper curve of the venous course (Fig 2). Technical success of the frontier-wire technique was defined as access into the involved CS compartment with the microcatheter through the occluded IPS and deployment of at least 1 coil into the target compartment.

At the end of the procedure, final angiographic results were classified as complete occlusion (no residual shunt), near-complete occlusion (small residual shunt with a marked reduction in 


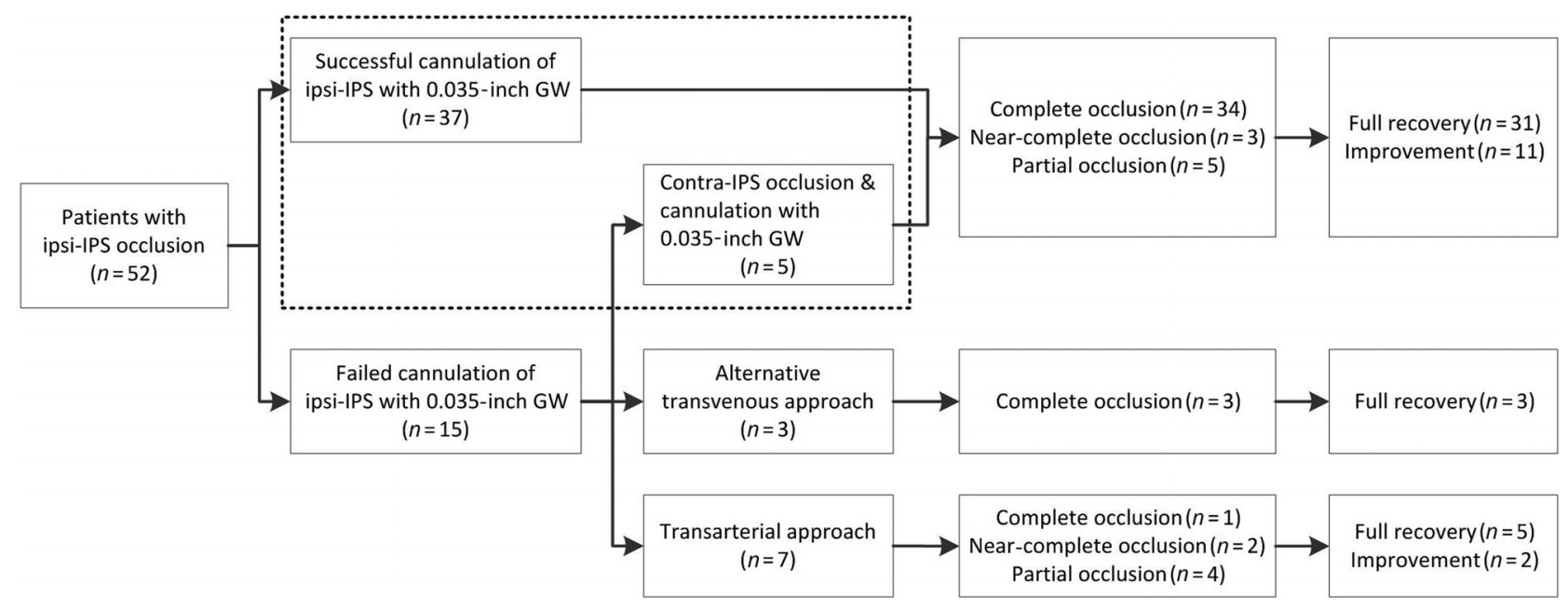

FIG 3. Flowchart for initial cannulation of the occluded ipsilateral-IPS. Patients within the dashed line had successful IPS cannulation. Ipsiindicates ipsilateral; Contra-, contralateral; GW, guidewire.

volume), and partial occlusion (large residual shunt with slight reduction). ${ }^{2}$ To obviate additional cranial nerve palsy related to mass effect or the coil mass, we tried to minimize coil volume as much as possible. We defined procedural success when the final angiography showed complete or near-complete occlusion of the shunt.

Clinical follow-up occurred at 1 and 3 months for patients with completely occluded fistulas. MR angiography was performed in patients with residual symptoms or residual shunts. Follow-up with digital subtraction angiography was performed selectively in patients with substantial residual symptoms or signs or an aggravated clinical condition. Clinical outcomes were classified as full recovery, improvement, or no change/aggravation.

\section{Statistical Analysis}

Baseline characteristics were summarized for patient groups as a number (percentage) for categoric variables and as the mean \pm SD for continuous variables. Differences were compared using the $t$ test for continuous variables, and the $\chi^{2}$ test or Fisher exact test for categoric variables. All reported probability values were 2-sided, and a probability value.

\section{RESULTS}

Cannulation of the occluded ipsilateral IPS using the frontierwire technique was attempted in all 52 patients and was successful in 37 patients. Cannulation of the occluded contralateral IPS was attempted and was successful in 5 patients (Fig 3 ). Accordingly, the technical success rates of the frontier-wire technique were $73.7 \%(42 / 57)$ of occluded IPSs and $80.8 \%$ (42/52) of patients.

The 0.035 -inch guidewire could be successfully advanced over the upper curve of the occluded vein in 45 of the 57 IPSs we attempted to cannulate, and technical success was achieved in 39 of the $45(86.7 \%)$. However, the 0.035 -inch guidewire could not be advanced over the upper curve in the other 12 occluded IPSs. Of those 12, we could advance the microcatheter system into the involved CS compartment in only 3 IPSs (25\%). Therefore, advancement of the guidewire over the upper curve was associated with a higher technical success rate $(P<0.001)$.

Due to failure to access the lesion even through the contralat- eral occluded IPS, alternative transvenous routes were used in 3 patients, including 1 patent contralateral IPS, 1 facial vein, and 1 superior ophthalmic vein with direct exposure. Thus, 45 patients underwent TVE. Complete occlusion was achieved in 37 patients; near-complete occlusion, in 3 patients; and partial occlusion, in 5 patients (88.9\% procedural success rate). Four patients underwent additional transarterial embolization of external carotid artery feeders using polyvinyl alcohol particles $(n=3)$ and glue $(n=1)$. Postprocedural symptom improvement was observed in all 45 patients (Fig 3).

Transarterial access was performed in 7 patients, and only external carotid artery feeders were selected and embolized (Fig 3). Single embolic material polyvinyl alcohol particles were used in 5 patients, coils were used in 1, and glue embolization with coils was used in 1 patient. Complete occlusion was achieved in 1 patient; near-complete occlusion, in 2 patients; and partial occlusion, in 4 patients. One patient underwent a second transarterial embolization, and 1 patient underwent radiation surgery for a residual fistula. Postprocedural symptom improvement was observed in all 7 patients. No procedure-related complications were observed in any of the 52 patients.

\section{DISCUSSION}

Although most patients with CSDAVFs present with benign neuro-ophthalmic symptoms, patients with higher risk CSDAVFs (such as fistulas with cortical venous drainage or hemorrhage) and patients with intolerable symptoms (such as diplopia, severe headache, or severe cosmetic disfigurement) usually require endovascular treatment to occlude the abnormal shunting of blood. ${ }^{1,2}$ TVE is regarded as the first-line treatment, and the ipsilateral IPS is usually the favored access route, whether the IPS is occluded or patent. ${ }^{2-4,8}$ The rate of successful catheterization of an occluded IPS with conventional methods varies but is reported to be approximately $54.3 \% .{ }^{3}$ Though Cho et $\mathrm{al}^{4}$ reported a microguidewire looping technique for breaching the ipsilateral IPS with a technical success rate of $80.0 \%(8 / 10)$, navigation through the invisible, obliterated IPS with a microcatheter and microguidewire still remains a challenging barrier. 
In this study, we present the frontier-wire technique using a 0.035-inch guidewire as a reproducible and robust method for cannulating the occluded IPS. A similar method was described in 1 case report ${ }^{3}$; however, the efficacy and safety of this approach have not been established in a larger study population. The physical basis of better performance of a guidewire thicker than a microguidewire system could be the following: 1) better controllability, 2) better pushability, and 3) the nature of the venous sinus obliteration. Because the occluded IPS is invisible on fluoroscopy, a blind manipulation procedure is necessary. ${ }^{3}$ Therefore, detailed knowledge of the anatomy of the IPS is essential for the effective and safe cannulation of an occluded IPS.

The IPS begins in the posteroinferior part of the CS; courses along the medial-lateral, anteroposterior, and rostral-caudal planes; and ends in the IJV. ${ }^{9}$ It is situated in the inferior petrosal sulcus, which is formed by the junction of the petrous part of the temporal bone with the basilar part of the occipital bone., ${ }^{9,10}$

To perform TVE through a thrombosed IPS, one must first identify the orifice of the IPS. ${ }^{3,8}$ According to a $3 \mathrm{D}$ rotational venography study, the drainage patterns of the IPS can be classified into the following 6 types based on the level of the IPS-IJV junction ${ }^{5}$ : The IPS drains into the jugular bulb (type A, 1.2\%); the IPS drains into the IJV at the level of the extracranial opening of the hypoglossal canal (type B, 34.9\%); the IPS drains into the lower extracranial IJV (type C, 37.3\%); the IPS forms a plexus and has multiple junctions to the IJV around the jugular foramen (type D, 6.0\%); the IPS drains into the vertebral venous plexus with no connection to the IJV (type E, 3.6\%); and the IPS is absent (type F, 16.9\%). Thus, types B and C are most common and should be considered first.

In the current study, we relied on only the anatomic knowledge and tactile sense of the operator to identify the IPS with 2D images of a biplane imaging system. Despite the high technical success rate, we may have failed in some difficult cases due to anatomic variations (such as no connection between the IPS and the IJV or an extremely low IPS orifice). ${ }^{5,11}$ Some alternative methods may be useful in such situations. Srivatanakul et $\mathrm{al}^{3}$ used $3 \mathrm{D}$ venography of the IJV to identify the remnant of the IPS. The best working angle for entering the IPS was found, and catheterization of the occluded IPS was performed by analyzing the 3D volume-rendering image. Yamauchi et $\mathrm{al}^{8}$ reported the usefulness of intravascular ultrasonography for detecting the caudal end of occult IPSs in patients with CSDAVFs.

Over the course of the IPS, there are many anastomotic channels among the IPS, the basilar venous plexus, the vertebral venous plexus, and the epidural venous plexus. ${ }^{11}$ The anastomotic channels connecting the IPS to the vertebral venous plexus (such as the anterior condylar vein) usually form an acute angle with the IPS, so the probing guidewire will usually not enter the anastomotic channels easily. However, in our experience, the probing guidewire tip could easily lodge in the anastomotic channels of the basilar venous plexus, which course in a direction similar to that of the IPS. ${ }^{12}$ Therefore, the guidewire tip should be turned upward to adapt to the course of IPS at its upper curve (Fig 2) and to avoid entering the anastomotic channels. Accordingly, we found that the passage of the upper curve with the probing guidewire is associated with a higher technical success rate of reaching the CS with the microcatheter.

There could be a psychological disinclination to perform blind manipulation with a regular 0.035 -inch guidewire through the IPS. However, in our study, no IPS perforation was observed. Hemorrhagic complications are also rare according to the medical literature on TVE of CSDAVFs; only 2 IPS perforations and 1 clival dura dissection were reported. ${ }^{1,13,14}$ In our experience, gentle manipulation of the guidewire based on the knowledge of IPS anatomy can effectively avoid perforation. If the guidewire goes medially and encounters resistance, the operator should be aware of the potential for injury to the basilar venous plexus and pull back the guidewire to probe another potential route. If there is substantial resistance when advancing the wire, switching to a microguidewire and a microcatheter is also a sensible alternative. If IPS perforation occurs, coil embolization may control the bleeding without difficulty due to the low pressure in the venous sinus. ${ }^{1}$ Furthermore, unlike bleeding from the artery, subarachnoid hemorrhage of venous origin secondary to sinus or dural injury may not require any further manipulation as long as the operator does not re-attempt cannulation through the injured IPS.

There are several limitations of this study. First, due to its retrospective nature, we could not distinguish the technical performance of the frontier-wire technique and the commonly used method with a microguidewire and a microcatheter. There could be a certain group enjoying successful cannulation with those microsystems. Second, 3D venography of the IJV was not performed to show the IPS-IJV junction anatomy; thus, we cannot correlate any anatomic variations to the causes of technical failure in this study. Third, as was seen in our report, successful cannulation of the IPS did not always guarantee a procedural success. Further morphologic analyses would help obviate unnecessary cannulation of the occluded IPS requiring an alternative treatment approach.

\section{CONCLUSIONS}

TVE of CSDAVFs, even through a completely occluded IPS, is feasible. The difficulty of passing a microcatheter can be minimized by prior probing of the occluded IPS using a standard 0.035 -inch guidewire; the trace of the guidewire can serve as a guide for microcatheter navigation through the IPS on fluoroscopy. Further study of IPS-IJV junction anatomy in difficult cases is needed to enhance the success rate of the frontier-wire technique.

\section{REFERENCES}

1. Kim DJ, Kim DI, Suh SH, et al. Results of transvenous embolization of cavernous dural arteriovenous fistula: a single-center experience with emphasis on complications and management. AJNR Am J Neuroradiol 2006;27:2078-82 Medline

2. Rhim JK, Cho YD, Park JJ, et al. Endovascular treatment of cavernous sinus dural arteriovenous fistula with ipsilateral inferior petrosal sinus occlusion: a single-center experience. Neurosurgery 2015; 77:192-99; discussion 199 CrossRef Medline

3. Srivatanakul K, Osada T, Aoki R, et al. Transvenous embolization of cavernous sinus dural arteriovenous fistula through a thrombosed inferior petrosal sinus utilizing 3D venography. Interv Neuroradiol 2015;21:362-65 CrossRef Medline 
4. Cho YD, Rhim JK, Yoo DH, et al. Transvenous microguidewire looping technique for breach of ipsilateral inferior petrosal sinus occlusions en route to cavernous sinus dural arteriovenous fistulas. Interv Neuroradiol 2016;22:590-95 CrossRef Medline

5. Mitsuhashi Y, Nishio A, Kawahara S, et al. Morphologic evaluation of the caudal end of the inferior petrosal sinus using 3D rotational venography. AJNR Am J Neuroradiol 2007;28:1179-84 CrossRef Medline

6. Mounayer C, Piotin M, Spelle L, et al. Superior petrosal sinus catheterization for transvenous embolization of a dural carotid cavernous sinus fistula. AJNR Am J Neuroradiol 2002;23:1153-55 Medline

7. Quiñones D, Duckwiler G, Gobin PY, et al. Embolization of dural cavernous fistulas via superior ophthalmic vein approach. AJNR Am J Neuroradiol 1997;18:921-28 Medline

8. Yamauchi S, Nishio A, Takahashi T, et al. An innovative technique for detecting the caudal end of occluded inferior petrosal sinus in cavernous arteriovenous fistula using intravascular ultrasonography: technical note. Neuroradiology 2015;57:799-804 CrossRef Medline
9. Ayeni SA, Ohata K, Tanaka K, et al. The microsurgical anatomy of the jugular foramen. J Neurosurg 1995;83:903-09 CrossRef Medline

10. Lo WW, Solti-Bohman LG. High-resolution CT of the jugular foramen: anatomy and vascular variants and anomalies. Radiology 1984;150:743-47 CrossRef Medline

11. Miller DL, Doppman JL, Chang R. Anatomy of the junction of the inferior petrosal sinus and the internal jugular vein. AJNR Am J Neuroradiol 1993;14:1075-83 Medline

12. Mortazavi MM, Griessenauer CJ, Krishnamurthy S, et al. The inferior petrosal sinus: a comprehensive review with emphasis on clinical implications. Childs Nerv Syst 2014;30:831-34 CrossRef Medline

13. Oishi $\mathrm{H}$, Arai $\mathrm{H}$, Sato K, et al. Complications associated with transvenous embolisation of cavernous dural arteriovenous fistula. Acta Neurochir (Wien) 1999;141:1265-71 CrossRef Medline

14. Luo CB, Chang FC, Teng MM, et al. Transvenous embolization of cavernous sinus dural arteriovenous fistula via angiographic occlusive inferior petrous sinus. J Chin Med Assoc 2015;78:526-32 CrossRef Medline 